PREPARED FOR THE U.S. DEPARTMENT OF ENERGY, UNDER CONTRACT DE-AC02-76CH03073

PPPL-3643

PPPL-3643

UC-70

Evaluation of Negative-Ion-Beam Driver

Concepts for Heavy Ion Fusion

by

Larry R. Grisham

January 2002

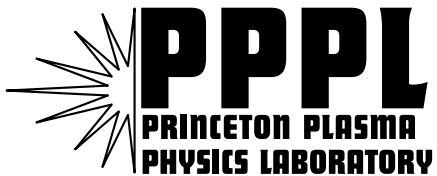

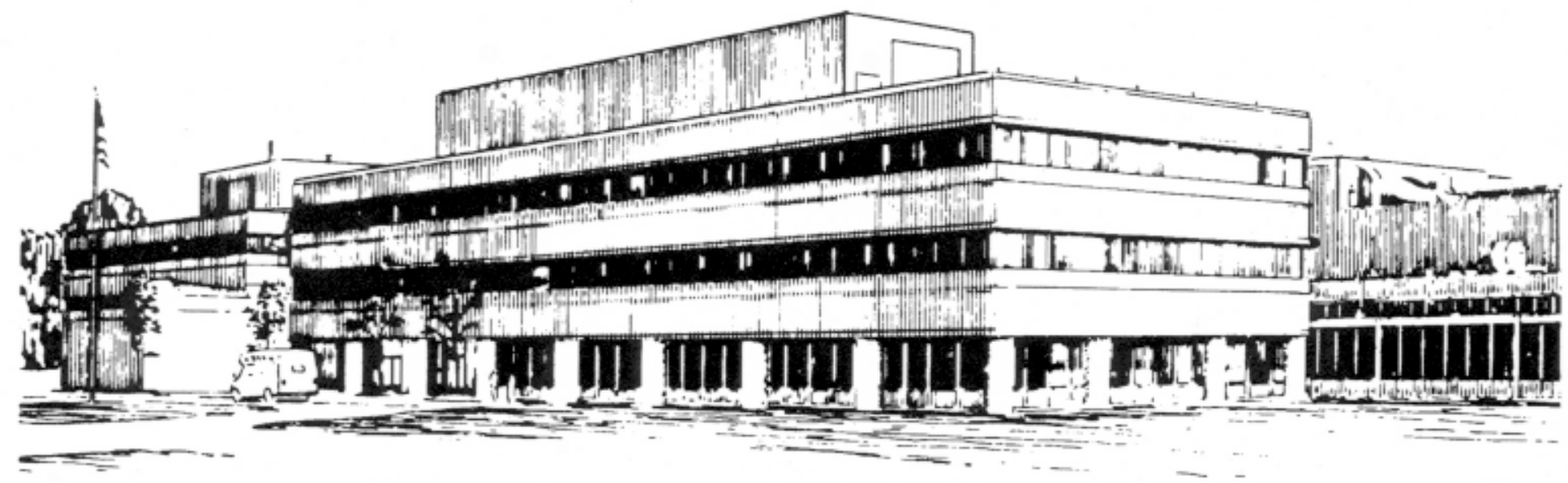

PRINCETON PLASMA PHYSICS LABORATORY PRINCETON UNIVERSITY, PRINCETON, NEW JERSEY 


\section{PPPL Reports Disclaimer}

This report was prepared as an account of work sponsored by an agency of the United States Government. Neither the United States Government nor any agency thereof, nor any of their employees, makes any warranty, express or implied, or assumes any legal liability or responsibility for the accuracy, completeness, or usefulness of any information, apparatus, product, or process disclosed, or represents that its use would not infringe privately owned rights. Reference herein to any specific commercial product, process, or service by trade name, trademark, manufacturer, or otherwise, does not necessarily constitute or imply its endorsement, recommendation, or favoring by the United States Government or any agency thereof. The views and opinions of authors expressed herein do not necessarily state or reflect those of the United States Government or any agency thereof.

\section{Availability}

This report is posted on the U.S. Department of Energy's Princeton Plasma Physics Laboratory Publications and Reports web site in Fiscal Year 2002. The home page for PPPL Reports and Publications is: http://www.pppl.gov/pub_report/

DOE and DOE Contractors can obtain copies of this report from:

U.S. Department of Energy

Office of Scientific and Technical Information

DOE Technical Information Services (DTIS)

P.O. Box 62

Oak Ridge, TN 37831

Telephone: (865) 576-8401

Fax: (865) 576-5728

Email: reports@adonis.osti.gov

This report is available to the general public from:

National Technical Information Service

U.S. Department of Commerce

5285 Port Royal Road

Springfield, VA 22161

Telephone: 1-800-553-6847 or

(703) 605-6000

Fax: (703) 321-8547

Internet: http://www.ntis.gov/ordering.htm 


\title{
Evaluation of Negative-Ion-Beam Driver \\ Concepts For Heavy Ion Fusion
}

\author{
Larry R. Grisham \\ Plasma Physics Laboratory \\ Princeton University, Princeton, NJ 08543
}

\begin{abstract}
We evaluate the feasibility of producing and using atomically neutral heavy ion beams produced from negative ions as drivers for an inertial confinement fusion reactor. Bromine and iodine appear to be the most attractive elements for the driver beams. Fluorine and chlorine appear to be the most appropriate feedstocks for initial tests of extractable negative ion current densities. With regards to ion sources, photodetachment neutralizers, and vacuum requirements for accelerators and beam transport, this approach appears feasible within existing technology, and the vacuum requirements are essentially identical to those for positive ion drivers except in the target chamber. The principal constraint is that this approach requires harder vacuums in the target chamber than do space-charge-neutralized positive ion drivers. With realistic (but perhaps pessimistic) estimates of the total ionization cross section, limiting the ionization of a neutral beam to less than $5 \%$ while traversing a four-meter path would require a chamber pressure of no more than $5 \times 10^{-5}$ torr. Alternatively, even at chamber pressures that are too high to allow propagation of atomically neutral beams, the negative ion approach may still have appeal, since it precludes the possibly serious problem of electron contamination of a positive ion beam during acceleration, drift compression, and focusing.
\end{abstract}




\section{Introduction}

A few years ago, we suggested that it might be feasible to use atomically neutral beams (as opposed to beams which are space-charge-neutralized) as the heavy particle driver beams for inertial confinement fusion (1). This approach would require producing heavy negative ions, accelerating them, and then converting them to neutral atoms with a photodetachment neutralizer just after the final focusing element, so that the beam traversing the target chamber would, along much of its path, be composed primarily of neutral atoms on ballistic targets.

A beam of energetic neutral atoms would not be subject to the vagaries of spacecharge forces, self-magnetic fields, or beam-plasma instabilities. This would greatly simplify the physics of beam transport in the target chamber, and allow a more compact and predictable final focus footprint. From the outset, it was appreciated that this approach would only be attractive for scenarios in which the target chamber operated at relatively hard vacuum, with accelerators at very hard vacuum. The impact of multielectron-loss processes $(\mathbf{1 , 2 , 3})$ would be as detrimental for atomically-neutral beams as for positive ion beams.

It was pointed out (4) that, even if one did not photodetach the negative ions because of inadequately low target chamber pressures, there might still be some merit to the use of negative ions inasmuch as negative ion beams would not draw electrons from the chamber plasma or from surfaces into the beam as it traversed the accelerator, drift compression region, and final focus optics.

In this paper, we describe a study to assess the viability of the negative-ion driver and neutral beam approach by considering the main critical issues. We also suggest a small experiments that could be carried out to test some aspects of the required technology. 


\section{Critical Technical Issues}

The critical technical issues for the negative-ion neutral beam approach are:

(1) Choice of beam element,

(2) Ion source,

(3) Photodetachment neutralizer,

(4) Vacuum requirements in accelerator and other parts of beam transport system, and

(5) Ionization of beam particles in the target chamber.

We briefly discuss these issues in the following sections of this paper.

\section{Choice of the Beam Element}

In order to produce negative ions, one must choose an element which has a finite electron affinity, which is the binding energy of the extra electron. In general, the greater the electron affinity, the easier it is to produce a negative ion of that element, and the more robust it is against being converted back to a neutral atom.

Throughout the periodic table, there are a number of elements with electron affinities of sufficient magnitude that they should be able to yield current densities of reasonable levels (10's - 100 or more per square-cm). Table I displays the atomic number, mass, and electron affinity of candidate beam elements. We have divided these candidates into three groups.

The first group should be relatively easy to make. These elements, mainly halogens, have large electron affinities, which means that a sizeable fraction (tens of percent) of the ions in most plasma discharges will naturally be negative ions, and it is probably possible to produce them all, if desired, from feedstocks that are either gases (fluorine and chlorine), elevated temperature vapors (bromine and iodine), or compound gases (such as hydrogen sulfide). Because negative ions produced from these elements will 
be relatively strongly bound, they are somewhat more robust in transport than negative ions produced from elements with lower electron affinities. However, this also means that higher-energy photons are required in the photodetachment neutralizer.

The second group of candidates consists of elements, mostly alkalis, whose lower electron affinities allow them to be neutralized by lower energy photons. This allows a wide choice among different types of lasers for a photodetachment neutralizer, since most lasers operate at photon energies greater than the binding energies of this group of negative ions. Correspondingly, the lower electron affinities of these elements mean that plasma discharges composed of these elements would contain a much lower fraction of negative ions than would be the case for the first group of candidate elements. Although not a heavy element, hydrogen, which has an electron affinity of $0.75 \mathrm{eV}$, is included for comparison because there is a great body of work connected with hydrogen negative-ion sources for magnetic confinement fusion applications. In these ion sources, the hydrogen negative-ion current which can be extracted from a source is usually only $2-3 \%$ of the positive-ion current which could be extracted under the same discharge conditions. Addition of cesium allows the negative-ion current to reach as much as $10-14 \%$ of the positive-ion current extractable under the same arc conditions. The mechanism of this enhancement remains somewhat obscure and controversial, and its applicability to other candidate beam elements is unclear, and unnecessary for the halogens. If, as discussed later, adequate laser technologies exist to photodetach the more tightly bound negative ions produced from the first group of elements, then this second group seems relatively unattractive because of the likelihood of lower negative-ion current densities.

The third group of candidate elements is comprised of heavy metals with a range of electron affinities. These seem unlikely to prove attractive because, while it is common to produce negative ions of at least some of them from sputter sources, it is not apparent how one would produce these by any means other than sputter sources. The negative ions produced by sputter sources typically have large transverse energies of at least several eV. This would probably preclude their use in heavy ion fusion, unless some way could be 
found around this technical issue.

The first two groups of candidate beam elements each span a fairly wide range of masses: $19-127$ for the first group, and $23-133$ for the second group. This allows some leeway in choosing an element, depending on how the heavy ion fusion program strategy evolves with the tradeoffs between mass, energy, current, and accelerator length.

\section{Ion Sources}

Plasma sources, similar to those used in magnetic confinement fusion, and driven either by arcs, RF, ECH, microwaves, or inductively, could be used to produce beams from all the candidate elements in Groups 1 and 2. In the cases of fluorine and chlorine, this should be especially straightforward, since these elements are naturally gases at room temperature. They are, of course, both poisonous, but no more so than feedstock gases which are commonly used in the semiconductor industry. More significantly, these, like most of the other candidate elements, are corrosive, which requires the use of appropriate materials.

Negative ions of sulfur could also be produced in a straightforward manner with conventional sources by using a gaseous feedstock such as hydrogen sulfide. Unfortunately, this would probably result in a number of negative-ion species being produced, since hydrogen has an appreciable electron affinity. Sulfur has a larger electron affinity, which should result in more negative ions of sulfur than of hydrogen. However, since the hydrogen negative ions are lighter and would have a greater drift velocity toward the extraction plane, the resulting beam might well consist of more hydrogen than sulfur.

Bromine, iodine and (perhaps) sulfur from the first group of candidate elements, and cesium, rubidium, potassium, and sodium from the second group could all be heated to a vapor in order to feed plasma sources operating at elevated temperatures. While conceptually straightforward, and almost certainly practical, this would be more complicated than simply changing the feedstock of an existing source to fluorine or 
chlorine. Thus, while the most desirable candidate elements for an HIF driver beam are bromine (mass 81 ) or iodine (mass 127) if an intermediate mass beam is wanted, it would be easier to initially test the ease of negative-ion production by introducing fluorine or chlorine gas into sources being tested for positive ions in the heavy ion fusion program. This would also require reversing the polarity of the extractor, and any pre-accelerator stage on the source, and changing the polarity of the grounding of the power supply. In addition, permanent magnets would need to be added across the extractor to suppress electrons. This is straightforward on a small system.

A simple test of this type with fluorine or chlorine should be a meaningful proof-ofprinciple experiment with respect to estimating the extractable negative-ion current densities (after adjusting for the differences in masses) which could be obtained using bromine and iodine. This is because the electron affinities of fluorine $(3.45 \mathrm{eV})$ and chlorine $(3.61 \mathrm{eV})$ are very similar to the electron affinity of bromine $(3.63 \mathrm{eV})$, and only modestly greater than that of iodine $(3.06 \mathrm{eV})$.

Because of the high electronegativity and large mass of bromine and iodine, their extractable current density will probably be limited by the extraction field strength that can be applied without breakdown occuring, rather than by the density of negative ions available, and thus will be a characteristic of the extractor design, rather than the polarity of the ion. Accordingly, for these ions of masses likely to be usable as HIF drivers, the extractable current density of negative ions may be essentially the same as would be obtainable with positive ions of similar masses.

In general, any type of source which produces positive ions from a plasma should be capable of producing negative ions with the right feedstock. If there is interest in this approach, it is appropriae that plasma sources being considered for positive ions for HIF be tested also with fluorine or chlorine. For a given type of source, the rise times and fall times of the extracted pulses should be similar for negative ions and positive ions. These time scales are primarily determined by the types of switches used in the circuits for the extractor and preaccelerator gaps. 
Negative-ion beams, especially when they are at low energy, are susceptible to stripping by gas along their flight path, which reduces them to neutrals or positive ions. This problem is most familiar with large hydrogen negative-ion sources, where the large extraction areas preclude the effective side pumping of the extractor and accelerator structure. This problem should be less severe for the cases we are interested in. Because the electron affinities of fluorine, chlorine, bromine, and iodine are all more than four times larger than that of hydrogen, the ratio of negative ions to neutral vapor propagating down the extractor and preaccelerator will be more favorable. In addition, the ions, being correspondingly more strongly bound, will be more robust. Since the ion source units will probably be considerably smaller than the hydrogen negative-ion sources presently in use in magnetic confinement fusion, the side pumping of the extractor and pre-accelerator should also be more practical. If bromine or iodine negative ions (or any of the alkali metals) are eventually produced from heated vapors, it will be necessary to also heat the grids and insulators in the extractor and preaccelerator to avoid metal vapor deposition and a corresponding compromise of voltage-holding characteristics.

\section{Photodetachment Neutralizers}

The neutralizer cells that are used in present day hydrogen negative-ion-based neutral beam systems to heat magnetic confinement fusion experiments use gas to collisionally detach the extra electron from a negative ion. However, multiple collisions can remove the remaining electron and produce a positive ion, so the neutralizer cell length has to be optimized. In practice, the maximum fraction of a hydrogen negative ion beam which can be converted to neutrals by a gas cell is about $60 \%$.

For heavier elements, gas cells are entirely impractical as neutralizers, due to the prevalence of multi-electron-loss events, which produce a wide range of charge states $(2,5,6)$. Using photons instead of gas to remove the extra electron from a negative ion sidesteps this problem. If the photon energy is a little greater than the binding energy of the 
extra electron (the electron affinity of the element), but less than the binding energy of the next electron (which is generally several times greater than the binding energy of the extra electron), then no positive ions will be produced (except through multiple photon absorption which is very rare). Accordingly, it is in principle possible to approach $100 \%$ efficiency in converting the negative ions to neutral atoms.

For this to be appealing, the energy used to drive the source of photons (usually a laser except in the case of very weakly bound negative ions) should be small compared to the energy in the extra fraction of neutral beam produced. Decades ago, photodetachment neutralizers were considered for the magnetic confinement fusion program, and again a decade ago for the neutral particle beam component of the Strategic Defense Initiative. In both cases, these approaches foundered upon two characteristics of the ion beams they were meant to neutralize: ion beam power density and pulse length.

The power density of the ion beams in the neutralizer cell for both of these applications was relatively low (a few kilowatts per square-cm), while the beam pulse length was long (essentially continuous for the magnetic confinement fusion application, and significant fractions of a second, with a very high duty factor, for defense applications). The low power density meant that it was difficult to use less power to drive the lasers than was gained in the extra neutralized beam, and the large cross-section of the beam required high laser power. The long or continuous pulse length meant that one was limited to lasers with continuous or near continuous capability, and thus to power levels orders-of-magnitude below those available with pulsed lasers.

For the case of heavy ion fusion, the conditions are much more favorable to photodetachment lasers. The power density in the ion beam is many orders-of-magnitude greater, and the pulse length and duty factor are millions of times less, permitting the use of pulsed laser technology.

Although the data base for photodetachment cross sections is relatively small, the cross-section generally rises steeply at photon energies just slightly greater than the binding energy, and then varies weakly with photon energies more than $0.2-0.4 \mathrm{eV}$ above the 
binding energy. Thus, for the more weakly bound alkali negative ions of the second group of candidate elements (with electron affinities of $0.47-0.54 \mathrm{eV}$ ), wavelengths shorter than about 1.9 microns should be usable. This rules out carbon dioxide, carbon monoxide, and holmium lasers, but leaves a wide range of possible choices, including, among others, iodine, Nd-glass YAG, color center, vibronic (Ti Sapphire), ruby, Kr-F, and Xenon lasers.

However, as discussed in the earlier sections, the negative ions which are most likely to produce sufficiently large current densities for a heavy ion driver are the more strongly bound halogens of the first group of candidate elements. With electron affinities of $3.06-3.63 \mathrm{eV}$, these require wavelengths shorter than 0.34 microns (for the most bound ion). According to the NRL Plasma Formulary (2000 edition), two laser systems capable of this are $\mathrm{Kr}-\mathrm{F}$ and Xenon. According to the Formulary, the state-of-the-art pulsed-power levels available around 1990 were $>10^{9}$ watts for the Kr-F laser, and $>10^{8}$ watts for the Xenon laser. For the same period, the state-of-the art efficiency of the Kr-F laser was 0.08, and of the Xenon laser 0.02. Both of these power levels and efficiencies should be acceptable for the application here.

Although the exact amount of laser power required would depend upon the detailed design of the neutralizer cell, the reflectivity of its mirrors, and the cross-section of the ion beam at the point where it is neutralized after the final focus elements, we can obtain an idea of whether the existing laser technology is likely to be adequate by considering a simple example.

Consider a negative-ion beam pulse with a cross section of 1 square-cm as it passes through the neutralizer cell, and with a pulse duration of 20 nanoseconds to neutralize a 10 nanosecond beam pulse. Although we don't presently have data on the photodetachment cross-sections for the beams we would most like to use, calculations and data for a variety of other negative ions in Massey (7), show photodetachment cross-sections in the range of $1 \times 10^{-17} \mathrm{~cm}^{2}$ to $2.4 \times 10^{-16} \mathrm{~cm}^{2}$.

We choose, for the purpose of this example, the lower end of this range $\left(1 \times 10^{-17}\right.$ 
$\mathrm{cm}^{2}$ ), as a reasonable estimate of the photodetachment cross-section of iodine. A Kr-F laser, which produces $4.7 \mathrm{eV}$ photons, should be very well suited for photodetaching negative ions of iodine, which are bound by $3.06 \mathrm{eV}$.

The line density (LD) of $4.7 \mathrm{eV}$ photons required to neutralize a fraction $n_{\mathrm{f}}$ of a 4 $\mathrm{GeV}$ negative-ion beam of iodine, is given by the expression:

$$
\mathrm{LD}=6.02 \times 10^{8} \ln \left(1 /\left(1-\mathrm{n}_{\mathrm{f}}\right)\right) \text { watts } / \mathrm{cm}
$$

To neutralize $99 \%$ of the beam, the required line density is $2.77 \times 10^{9}$ watts $/ \mathrm{cm}$. For a 20 nanosecond pulse, the required laser energy is 55.4 joules/cm, and to maintain this line density across a beam diameter of $3 \mathrm{~cm}$, the required laser energy per pulse is 166.2 joules. For mirrors allowing 100 reflections, which should be readily available, this energy requirement would be reduced to about 1.7 joules, and the corresponding laser power is $2.77 \times 10^{7}$ watts $/ \mathrm{cm}$. In 20 nanoseconds, light travels 6 meters, which would be enough time for 150 transits along a $4 \mathrm{~cm}$ bounce path.

With a laser efficiency of 0.08 , a laser burst of 1.7 joules would require an input power of about 21 joules. This is two orders-of-magnitude less than the state-of-the-art for Kr-F lasers in about 1996.

There are some simplifications and uncertainties in this simple example; however, dielectric mirrors with reflectivities in excess of $99.9 \%$ have been available for many years, and designs have been carried out in the past for laser neutralizer cavities in which the beam was confined for several thousand bounces. Thus, one could gain another factor of 10 or much more with the correct choice of mirrors and cavity design. Accordingly, even a completely realistic and correct engineering design of the laser neutralizer should not change the conclusion that the laser technology requirements for a heavy ion photodetachment neutralizer are well within the state of existing technology. 
VI. Vacuum Requirements in the Accelerator and Beam Transport Regions

At low energies of a few tens of kev/amu, the cross-sections for neutralizing a highenergy negative-ion or converting are considerably larger than the cross-sections for converting a positive ion to a neutral. Thus, the vacuum requirements are more stringent for transporting and accelerating low-energy negative ions than for their positive counterparts.

However, once the ions reach energies of 100's of keV/amu to 10's of MeV/amu, the difference in vacuum requirements for positive and negative ions is likely to be much less significant. This is because, at higher energies, the positive ions are themselves subject to ionization to higher charge states, with total cross-sections that are probably not significantly smaller than for the negative ions. One can see this readily from the fact that the translational kinetic energy of the electrons is larger than the binding energies for most of the electrons in the projectile's electron cloud, not simply the extra electron of the negative ion. For example, at an energy of just $1.4 \mathrm{MeV} / \mathrm{amu}$, the translation of kinetic energy of the bound electrons is $0.76 \mathrm{keV}$.

The ions are most susceptible to destruction when they are at low energy in the region extending from the source and pre-accelerator to the first higher-energy accelerator. However, if the beam ion is iodine or bromine, the most likely choices for a negative-ion driver, then the feedstock will most likely be metal vapor, which is very easily pumped with cooled surfaces. Thus, since this region is short, it should be fairly easy to keep losses to an acceptable level prior to the start of the RF accelerator sequence.

A much more serious problem is the vacuum requirements for the vastly longer path length through the RF accelerators, drift-compression region, and final focus optics. As an example, we consider a path length of $1 \mathrm{~km}$, and we take the path-averaged total ionization cross-section to be $1.6 \times 10^{-16} \mathrm{~cm}^{2}$.

We arrive at this estimate for the cross-section in the following way. Reference ( 2 ) measured the total ionization cross-section for $3.4 \mathrm{MeV} / \mathrm{amu} \mathrm{Kr}^{+7}$ passing through 
molecular nitrogen to be $0.78 \times 10^{-16} \mathrm{~cm}^{2}$. This cross-section includes the cross-section for single ionization $\left(0.44 \times \mathrm{x}^{-16} \mathrm{~cm}^{2}\right)$ as well as the cross-section for multielectron loss events $\left(0.34 \times \times 10^{-16} \mathrm{~cm}^{2}\right)$. For-singly charged positive ions (which would be characteristic of drivers currently envisioned), the cross-section would be somewhat higher than this, and for negative ions, slightly higher still. On the other hand, heavy ion fusion driver beams will likely operate at 20 - $40 \mathrm{MeV} / \mathrm{amu}$ or higher, and these cross -sections should decline with energy. Consequently, we have chosen to double the experimentallymeasured cross-section as a reasonable estimate for this evaluation. This is meant to approximate the path-averaged destruction cross-section, from the beginning of the RF accelerator, where the energy is a few 10's of keV/amu, through the drift-compression region, where it is 10's of MeV/amu.

If the path-averaged destruction cross-section is $1.6 \times 10^{-16} \mathrm{~cm}^{2}$ over a path length of $1 \mathrm{~km}$, and if we lose less than $5 \%$ of the negative ions, then the pressure should be no higher than $1 \times 10^{-7}$ torr. For a system this large, this pressure is probably modestly challenging, but not intimidating. In any event, as discussed above, the high -energy vacuum requirement for negative ions should not differ significantly from whatever is determined to be necessary for singly charged positive ion beams.

Another source of ion charge state change is Lorentz ionization, which can occur when particles pass through strong magnetic fields. However, it takes considerable effort to make this happen even with very weakly bound negative ions such as $\mathrm{He}^{-}$, which has a binding energy of only $0.075 \mathrm{eV}$, so it should not be a significant problem for negative ions like fluorine, chlorine, bromine, or iodine, with their binding energies of $3.06-3.63$ $\mathrm{eV}$.

\section{Ionization of the Beam in the Target Chamber}

From the outset, it was fully appreciated that the most critical issue, and the one which would most severely limit the use of atomically neutral heavy ion beams as drivers, 
is the gas pressure in the target chamber for whatever scenario ends up being adopted for a fusion reactor.

To estimate the vacuum requirements in the target chamber, we again use the measurements of Reference (2). These measurements were done with molecular nitrogen as the target gas because it should be a good model of FLIBE vapor. The measurement of $0.78 \times 10^{-16} \mathrm{~cm}^{2}$ for the total ionization probability (including multielectron loss events) was done at $3.4 \mathrm{MeV} / \mathrm{amu}$ on $\mathrm{Kr}^{+7}$. The beams crossing the target chamber will be at higher energies of 20 - $40 \mathrm{MeV} / \mathrm{amu}$, which will reduce the cross-section, perhaps by as much as the energy ratio (which would be a factor of 6 - 12). However, the reduction might be weaker than this, due to the influence of multielectron loss events, because progressively more electrons become accessible to ionization until the translational kinetic energy of the innermost electrons exceeds their binding energy. On the other hand, the beam traversing the target chamber in this scenario will be atomically neutral, so the total ionization cross section will be larger at a given energy than the one measured in Reference (2).

Accordingly, we have attempted to balance these three opposing trends by making the probably conservative (i.e., pessimistic) assumption that the total ionization crosssection for 20 - $40 \mathrm{keV} / \mathrm{amu}$ neutral atom beams traversing FLIBE vapor in the target chamber is simply the $0.78 \times 10^{-16} \mathrm{~cm}^{2}$ value measured in Reference (2). If we assume a four- meter flight path from the photodetachment neutralizer across the target chamber medium to the target, and if we want to ionize less than $5 \%$ of the neutral beam, then the pressure should be no more than $5 \times 10^{-5}$ torr. This is a fairly stringent requirement, especially for a target chamber with liquid FLIBE walls and jets. The HYLIFE-II (8) reactor design was expected to have a pressure of $1.7 \times 10^{-3}$ torr of beryllium difluoride vapor. However, recent work suggests (9) that it should be possible to reduce this pressure by factors of 5 or more by various means, including using some lower temperature FLIBE jets to shield higher temperature flows, and by other measures with different salt mixtures (10). 


\section{Conclusions}

It appears that bromine and iodine offer the most attractive negative ions for heavy ion beam neutral-atom drivers. However, fluorine and chlorine will be the easiest gases to use for any initial tests of available negative-ion current densities from practical sources. Since their electron affinities are quite similar to those of bromine and iodine, it should be reasonable to extrapolate the current densities that could be produced with those ions from results obtained with fluorine and chlorine, after allowing for mass effects on the perveance.

It also appears that modifications of positive-ion source technology are likely to result in adequate negative-ion current densities from these halogens. The requirements for photodetachment neutralizers appear to be fairly moderate, and well within the state of the art. The negative ion pressure requirements on the accelerators, transport, focusing, and drift-compression regions should be almost identical to the pressure requirements for positive heavy ion beams. The one limiting constraint is the one initially anticipated, which is that for the atomic beams to reach the target as neutrals, the pressure in the target chamber needs to be low. For less than a 5\% ionization loss across a four-meter flight path, our estimate is that the pressure should be no higher than $5 \times 10^{-5}$ torr. This estimate uses assumptions about the cross-sections which are arguably pessimistic, but it is in any event clear that this scenario requires lower pressures than those presently being considered for space-charge-neutralized positive-ion driver beams. Work by Molvik et. Al. Suggests that significant reductions in chamber pressure are possible $(\mathbf{9 , 1 0})$.

A logical next step should be to try fluorine, or more probably, chlorine, as a feedstock in a candidate HIF positive-ion source (with the accelerating polarity reversed and with permanent magnets added to the grid for electron suppression) to give an experimental evaluation of extractable negative-ion current densities. If these results are favorable, then it could serve as the basis for the design of an elevated temperature ion 
source using bromine or iodine.

Even if scenarios for HIF target chambers do not ever return to versions with sufficiently low pressures to allow photodetached atomically neutral beams to propagate to the target without significant ionization, negative ion driver beams still have an advantage which might turn out to be crucial. Negative ion beams will not invite the accumulation of electrons pulled by strong electric fields from nearby surfaces which may be a problem for intense heavy positve ion beams passing through the long accelerator, drift compression space, and focusing elements. Unless this electron contamination is highly reproducible from shot to shot, it could alter the focusing in ways that might be very difficult to compensate. Thus, heavy negative ions such as bromine or fluorine could serve as a backup technology for the HIF program if electron contamination should turn out to be an intractable problem in positive ion beams.

\section{Acknowledgments}

This research was supported by the U.S. Department of Energy. It is a pleasure to acknowledge conversations with D. Callahan, J. Kwan, E. Lee, N. Leung, G. Logan, and A. Molvik. We appreciate the encouragement and support of R. Davidson and R. McKnight. 


\section{References}

[1] L. R. GRISHAM, "Possible Impact of Multi-electron Loss Events on the Average Beam Charge State in an HIF Target Chamber and a Neutral Beam Approach”, Nucl. Instr. Meth. Phys. Res. A 464, 315 (2001).

[2] D. MUELlER, L. GRISHAM, I. KAGANOVICH, R. WATSON, V. HORVATH K.ZAKARAS, and M. ARMEL, "Multiple Electron Stripping of $3.4 \mathrm{Mev} / \mathrm{amu} \mathrm{Kr}{ }^{7+}$ and $\mathrm{Xe}^{11+}$ in Nitrogen", Physics of Plasmas 8, 1753 (2001).

[3] I. D. KAGANOVICH, E. STARTSEV and R. C. DAVIDSON, "Evaluation of Multielectron Ionization Cross-sections in Energetic Ion-Atom Collisions", Proc. of Particle Accelerator Conference 2001, Chicago, (2001) URL: http://pacwebserver.fnal.gov/papers/Tuesday/AM_Poster/TPAH314.pdf

[4] ED LEE, private communication (1999).

[5] L. R. GRISHAM, D. POST, B. JOHNSON, K. JONES, J. BARETTE, T. KRUSE, I. TSERRUYA, and W. DA-HAI, "Efficiencies of Gas Neutralizers for Multi-MeV Beams of Light Negative Ions”, Princeton Plasma Physics Laboratory Report PPPL-1857 (1981).

[6] L. R. GRISHAM, D. POST, B. JOHNSON, K. JONES, J. BARRETTE, T. KRUSE, I. TSERRUYA, and W. DA-HAI, "Efficiencies of Gas Neutralizers for MultiMeV Beams of Light Negative Ions”, Rev. Sci. Instr. 53 no. 3, 281 (1982).

[7] H. S. W. MASSEY, Negative Ions ( $3^{\text {rd }}$ Edition, Cambridge University Press, 1976). 
[8] D. A. CALLAHAN, "Chamber Propagation Physics for Heavy Ion Fusion", Fus. Eng. \& Design 32-33, 441 (1996).

[9] A. W. MOLVIK, R. W. MOIR, C JANTZEN, and P. PETERSON, "Higher vacuum and faster clearing in a liquid-walled IFE chamber", Bull. Am. Phys. Soc., 45, 206 (2000).

[10] A. W. MOLVIK, private communication (2002). 
Table I

\section{Electron Affinities of Selected Elements}

Element $Z \quad$ Mass Electron Affinity $(\mathrm{eV})$

Group 1. Relatively Easy to Make:

$\begin{array}{llll}\mathrm{F} & 9 & 19 & 3.45 \\ \mathrm{Cl} & 17 & 37 & 3.61 \\ \mathrm{Br} & 35 & 81 & 3.63 \\ \mathrm{I} & 53 & 127 & 3.06 \\ \mathrm{~S} & 16 & 32 & 2.08\end{array}$

Group 2. Relatively Easy to Photodetach:

$\begin{array}{llll}\mathrm{Cs} & 55 & 133 & 0.47 \\ \mathrm{Rb} & 37 & 85 & 0.49 \\ \mathrm{~K} & 19 & 40 & 0.49 \\ \mathrm{Na} & 11 & 23 & 0.54 \\ \mathrm{H} & 1 & 1 & 0.75\end{array}$

Group 3. Require Sputter Sources:

$\begin{array}{llll}\mathrm{Sb} & 51 & 123 & 1.05 \\ \mathrm{Ag} & 47 & 109 & 1.30 \\ \mathrm{Au} & 79 & 197 & 2.31 \\ \mathrm{Pt} & 78 & 195 & 2.13\end{array}$




\section{External Distribution}

Plasma Research Laboratory, Australian National University, Australia

Professor I.R. J ones, Flinders University, Australia

Professor J oão Canalle, Instituto de Fisica DEQ/IF - UERJ , Brazil

Mr. Gerson O. Ludwig, Instituto Nacional de Pesquisas, Brazil

Dr. P.H. Sakanaka, Instituto Fisica, Brazil

The Librarian, Culham Laboratory, England

Library, R61, Rutherford Appleton Laboratory, England

Mrs. S.A. Hutchinson, JET Library, England

Professor M.N. Bussac, Ecole Polytechnique, France

Librarian, Max-Planck-Institut für Plasmaphysik, Germany

J olan Moldvai, Reports Library, MTA KFKI-ATKI, Hungary

Dr. P. Kaw, Institute for Plasma Research, India

Ms. P.J . Pathak, Librarian, Insitute for Plasma Research, India

Ms. Clelia De Palo, Associazione EURATOM-ENEA, I taly

Dr. G. Grosso, Instituto di Fisica del Plasma, Italy

Librarian, Naka Fusion Research Establishment, J AERI, J apan

Library, Plasma Physics Laboratory, Kyoto University, J apan

Research Information Center, National Institute for Fusion Science, J apan

Dr. O. Mitarai, Kyushu Tokai University, J apan

Library, Academia Sinica, Institute of Plasma Physics, People's Republic of China

Shih-Tung Tsai, Institute of Physics, Chinese Academy of Sciences, People's Republic of China

Dr. S. Mirnov, TRINITI, Troitsk, Russian Federation, Russia

Dr. V.S. Strelkov, Kurchatov Institute, Russian Federation, Russia

Professor Peter Lukac, Katedra Fyziky Plazmy MFF UK, Mlynska dolina F-2, Komenskeho Univerzita, SK-842 15 Bratislava, Slovakia

Dr. G.S. Lee, Korea Basic Science Institute, South Korea

Mr. Dennis Bruggink, Fusion Library, University of Wisconsin, USA

Institute for Plasma Research, University of Maryland, USA

Librarian, Fusion Energy Division, Oak Ridge National Laboratory, USA

Librarian, Institute of Fusion Studies, University of Texas, USA

Librarian, Magnetic Fusion Program, Lawrence Livermore National Laboratory, USA

Library, General Atomics, USA

Plasma Physics Group, Fusion Energy Research Program, University of California at San Diego, USA

Plasma Physics Library, Columbia University, USA

Alkesh Punjabi, Center for Fusion Research and Training, Hampton University, USA

Dr. W.M. Stacey, Fusion Research Center, Georgia Institute of Technology, USA

Dr. J ohn Willis, U.S. Department of Energy, Office of Fusion Energy Sciences, USA

Mr. Paul H. Wright, Indianapolis, Indiana, USA 
The Princeton Plasma Physics Laboratory is operated by Princeton University under contract with the U.S. Department of Energy.

\author{
Information Services \\ Princeton Plasma Physics Laboratory \\ P.O. Box 451 \\ Princeton, NJ 08543
}

Phone: 609-243-2750

Fax: 609-243-2751

e-mail: pppl_info@pppl.gov

Internet Address: http://www.pppl.gov 